\title{
New York Heart Association Class III/IV
}

National Cancer Institute

\section{Source}

National Cancer Institute. New York Heart Association Class IIIIIV. NCI Thesaurus. Code C7922.

The two most severe classes of cardiac dysfunction according to a classification system developed by the New York Heart Association to grade cardiovascular function and disability. These classes are defined as follows: Class III - patients with cardiac disease producing marked limitation of activity: comfortable at rest. Less than ordinary physical activity causes symptoms. Class IV - patients with cardiac disease resulting in inability to carry on any physical activity without discomfort. Symptoms may be present even at rest. 